\title{
COMPARATIVE ASSESSMENT OF ANTIBACTERIAL ACTIVITY AND FLUORIDE RELEASE POTENTIAL OF CENTION-N AND DIFFERENT BIOACTIVE RESTORATIVE MATERIALS: AN IN VITRO STUDY
}

\author{
Walaa Mohamed Alsamolly* and Galal El Deen Mosaad Sadek**
}

\begin{abstract}
Aim: This investigation was organized to assess the antibacterial action and fluoride release capacity of Alkasite material (Cention $\mathrm{N}$ ) in compare with two bioactive materials (Activa BioACTIVE restorative and Zirconomer) at different time intervals

Materials and methods: Total 75 disc formed specimens of 3 various restorative materials (Zirconomer, Activa BioACTIVE, and Cention N) were prepared. 45 specimens for fluoride release $(\mathrm{n}=15)$ and 30 specimens for antibacterial activity $(\mathrm{n}=10)$. For fluoride release the specimens divided into 3 groups $(\mathrm{n}=15)$ Group1: Zirconomer, Group2: Cention $\mathrm{N}$ and Group 3: Activea bioactive. Each fluoride release group was further subdivided into three equal subgroups $(n=5)$ according to the storage time (one day, 7days and 14days). For Antibacterial activity specimens divided into 3 groups ( $\mathrm{n}=10)$ Group1: Zirconomer, Group2: Cention N and Group 3: Activea bioactive. Fluoride release was estimated at the finale of Day 1, 7and Day 14, using fluoride ion-selective electrode. Also, Antibacterial Activity and diameters of inhibition zone were recorded after 48 hours. Data analysis was performed using ANOVA and post-hoctest.
\end{abstract}

Results: Zirconomer group revealed significantly the highest mean fluoride release value than in Cention $\mathrm{N}$ and Activea bioactive. Also, Cention $\mathrm{N}$ significantly higher fluoride release than Activa BioACTIVE. Cention N group showed statistically significant largest inhibition zone anti Streptococcus mutans at 48 hours followed by Zirconomer group, the smallest inhibition zone was observed with Activa bioactive group.

Conclusion: Cention $\mathrm{N}$ and Zirconomer had better antibacterial activity and fluoride release while Activea bioactive had limited antibacterial activity and fluoride release.

KEY WORDS: Bioactive restoration, Alkasite restoration, antibacterial activity, Fluoride release.

* Lecturer of Operative Dentistry, Faculty of Dentistry, Al-Azhar University (Boys) Cairo

** Lecturer of Dental Bio-material, Faculty of Dentistry, Al-Azhar University (Boys) Cairo. 


\section{INTRODUCTION}

Dental materials that have both restorative and anticariogenic features are ideal to control dental caries ${ }^{(1)}$. Fluoride is well recognized as an anticariogenic agent, its task involves decline demineralization, remineralization improvement, interference of plaque formation and inhibition of microbial growth. There are numerous materials that perform anticariogenic action by fluoride release. Glass Ionomer Cement is the commonly handled and significantly admired fluoride- releasing restorative material. ${ }^{(2)}$

Traditional glass ionomer has an advantage of fluoride liberation but its insufficient strength has restricted its employment in less stress-bearing regions. This drawback led to the necessity for recent materials with fluoride release, as compomers, giomers, and numerous innovative glass hybrid materials which was improved the strength, and overcome some of the drawbacks of traditional glass ionomer. ${ }^{(3)}$

Gradually, recent and enhanced materials for restorations have been introduced as Cention $\mathrm{N}$, activea Bioactive restorative and Zirconomer. ${ }^{(4)}$ Cention $\mathrm{N}$ is analogous to glass ionomer cement and resin modified glass ionomer where it has fluoride liberation and as well act as an aesthetic filling material. This material has reasonably more transparency when matched to other glassionomer products, hence getting improved aesthetic properties..$^{(4,5)}$ Additionally, it has enhanced compressive strength. In contrast to its equivalents, Cention $\mathrm{N}$ has more various uses. Cention $\mathrm{N}$ devours an alkaline filler which may deliver acidneutralizing particles. ${ }^{(4)}$

Cention - $\mathrm{N}$ has $78 \%$ by weight inorganic filler. The alkaline glass represents $24 \%$ in weight of the final material and this liberate significant degrees of fluoride particles like those liberate by traditional glass ionomer. ${ }^{(4)}$
An innovative category of bioactive restoratives (Activa-Restorative) in restorative dentistry has been introduced. ${ }^{(6)}$ Their producer claims to take the common properties of a resin modified glass ionomer with improved bioactive ionic resin matrix with improved elasticity and physical characters, it has both light curing ability and chemical cure and also have fluoride ions liberation potential. This restorative material is the earliest bioactive dental material with an ionic resin matrix and bioactive fillers that mimic the physical and chemical characters of natural teeth. ${ }^{(7,8)}$

Recently, zirconia reinforced glass ionomer cement has been introduced. it has the strength and longevity of amalgam with completely removing the risk of mercury. ${ }^{(9)}$ Zirconomer was developed as a reliable and durable self-adhesive tooth-colored zirconia-reinforced posterior bulk-fill restorative material. It comprises nano-sized zirconia fillers for superior handling characteristics and also to enhance esthetic properties ${ }^{(10)}$. The Polyalkenic acid and the glass components of Zirconomer have been specially processed to impart superior mechanical, fluoride release and handling qualities. ${ }^{(11)}$

Fluoride release from restorative materials during short and long-time span relies on numerous aspects such as nature and amount of fluoride incorporated, matrices of materials and setting responses ${ }^{(12)}$.

This examination was directed to assess the antibacterial effect of alkasite material (Cention $\mathrm{N}$ ) against Streptococcusmutans and its fluoride release potential at various time intervals in comparison with two different bioactive materials (Activa BioACTIVE and Zirconomer).

\section{MATERIALS}

Three distinctive restorative materials were utilized in this study as demonstrated in table (1). 
TABLE (1): Material category, brand name, composition, manufacture and batch number of the material used.

\begin{tabular}{|c|c|c|c|}
\hline Material Category & $\begin{array}{l}\text { Brand } \\
\text { Name }\end{array}$ & Composition & $\begin{array}{l}\text { Manufacture \& } \\
\text { batch number }\end{array}$ \\
\hline $\begin{array}{l}\text { Bioactive } \\
\text { restorative material }\end{array}$ & $\begin{array}{l}\text { ACTIVA } \\
\text { Bioactive }\end{array}$ & $\begin{array}{l}\text { Mixture of diuerthane and additional methacrylate with } \\
\text { enhanced polyacrylic acid. } 55 \text { wt. } \% \text { bioactive glass and } \\
\text { sodium fluoride. }\end{array}$ & $\begin{array}{c}\text { Pulpdent. Watertown, } \\
\text { MA. USA(151217) }\end{array}$ \\
\hline $\begin{array}{c}\text { Bioactive restorative } \\
\text { material }\end{array}$ & Zirconomer & $\begin{array}{l}\text { Powder: Fluor aluminosilicate glass, zirconium oxide, } \\
\text { colorants and others. } \\
\text { Liquid: polyacrylic and tartaric acid. }\end{array}$ & $\begin{array}{c}\text { Shofu.Kyoto, } \\
\text { Japan(03150682) }\end{array}$ \\
\hline $\begin{array}{l}\text { Alkasite } \\
\text { restorative material }\end{array}$ & Cention-N & $\begin{array}{l}\text { Powder: glass fillers (barium aluminium silicate glass filler, } \\
\text { ytterbium trifluoride, an Isofiller, a calcium barium aluminium } \\
\text { fluorosilicate glass filler and a calcium fluorosilicate (alkaline) } \\
\text { filler, initiator (Ivocerin) and pigments. } \\
\text { Liquid dimethacrylates (urethanedimethacrylate (UDMA), } \\
\text { an aromatic aliphaticUDMA) and initiators. }\end{array}$ & $\begin{array}{l}\text { Ivoclar Vivadent, } \\
\text { Schaan, } \\
\text { Liehtenstei(W94184) }\end{array}$ \\
\hline
\end{tabular}

\section{METHOD}

\section{Sample grouping:}

A total number of 75 specimens were fabricated using a specially constructed standardized split teflon mold to be packed with the different tested restorative materials. The specimens were divided according to the evaluation parameter into 45 specimens for fluoride release and 30 specimens for antibacterial activity.

\section{For fluoride release $(n=15)$}

Group 1. Zirconia reinforced glass ionomer (Zirconomer)

Group 2. Alkasite restorative material (Cention N)

Group 3. Bioactive restorative composite (Activea Bioactive restorative)

Each fluoride release group was additionally partitioned into three equivalent subgroups $(n=5)$ corresponding to the storage time (one day, 7days and 14 days).

\section{For Antibacterial activity $(n=10)$}

Group 1. Zirconia reinforced glass ionomer (Zirconomer)

Group 2. Alkasite restorative material (Cention N).

Group 3. Bioactive restorative composite (Activea Bioactive restorative)

\section{Sample Preparation}

Seventy-Five cylindrical disc formed specimens for fluoride releasing and antibacterial activity test were made in a standardized split Teflon mold ( $3 \mathrm{~mm}$ thickness and $6 \mathrm{~mm}$ diameter).

The Sterile split Teflon mold was put on the highest point of a sterile microscope glass slide and a celluloid strip, the restorative material samples were packed into the mold using sterile goldplated instrument, the second celluloid strip was used to overlay the top side of the mold to inhibit construction of oxygen inhibited layer. Additional microscope glass slide and $500 \mathrm{gm}$ weight were employed above the second celluloid strip for 30 s 
to give a consistent, good packing of the specimens and to extrude the excess material. Employed weight and microscope slide were eliminated, applied light for Polymerization was achieved be in harmony with manufacture suggestion utilizing light-curing device (Elipar S10, 3M ESPE; USA, wavelength $455 \mathrm{~nm} \pm 10 \mathrm{~nm}$, light intensity $1200 \mathrm{~mW} / \mathrm{cm}^{2}$ ). The tip of the light curing unit was kept focused in direct contact with the celluloid strips on the top surface of the mold perpendicular to it. Following photopolymerization the cylinder-shaped specimens were detached from their molds and washed with tap water for 1minute and then examined for their width and length using a digital caliper, all specimens were then polished Figure (1).

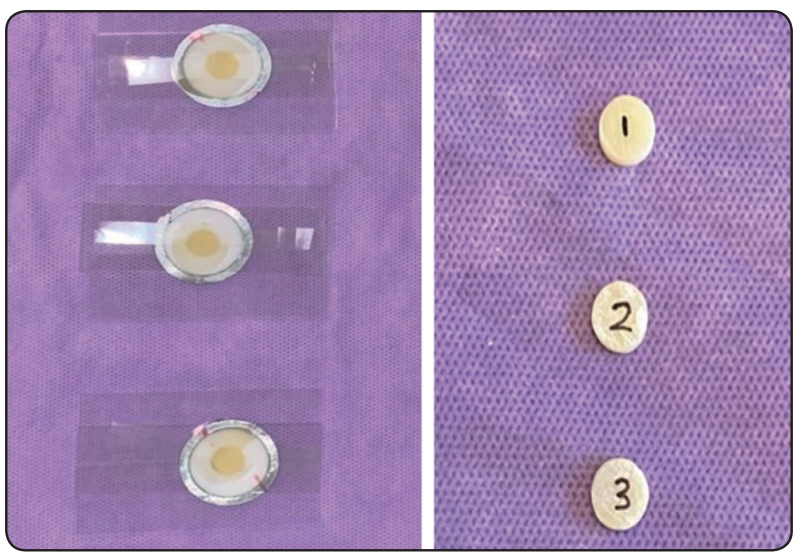

Fig. (1): Specimens after polymerization

\section{Storage of the specimens}

\section{Storage of the specimens for fluoride release test}

Every specimen was submerged in a plastic container having $5 \mathrm{~mL}$ of deionized water at $37^{\circ} \mathrm{C}$ (triple distilled water ANION $\mathrm{H}+\mathrm{CATION}$ $\mathrm{OH}=\mathrm{H}_{2} \mathrm{O}$ and free from any minerals) specially prepared in faculty of pharmacy, Cairo University.

After $24 \mathrm{~h}$, the containers were carefully shaken and the water eliminated and examined. The specimens were then reimmersed in $5 \mathrm{~mL}$ of new deionized water that was exchanged every $24 \mathrm{~h}$ for additional equilibration.
Fluoride release was studied at day one, day 7 and day 14 . Each $5 \mathrm{ml}$ storage water was blended with $0.5 \mathrm{~mL}$ of ionic strength adaptable buffer TISAB III (Total ionic strength regulation buffer solution, Germany) was further put in to monitor $\mathrm{pH}$ and to inhibit the development of fluoride complexes. The use of TISAB III liberates fluoride ions joined to hydrogen and removes hydroxyl ion interference, so allowing a perfect measurement of the total fluoride content.

The concentration of the fluoride ion was calculated after equilibration of the solution by a fluoride specific ion electrode (Fluoride Meter), and it was calibrated with multiple standard solutions of $0.1,1,10,50$ and 100ppm fluoride.

\section{Storage of the specimens for Antibacterial activity test}

Streptococcus mutans ATCC 25175 form strain was obtained from (Microbiological Resources Centre, Cairo, Egypt) and used throughout the study.

Bacteria were cultured overnight at $37^{\circ} \mathrm{C}$ in Brain Heart Infusion Broth (BHI, Oxoid, and Basingstoke, England) and used as inoculums. The inoculum $(100 \mu \mathrm{l})$ was regulated to equal the turbidity of 0.5 McFarland values, and was swabbed on Trypticase Soy agar and approved to dry for $10 \mathrm{~min}$.

Prepared Specimens of experimental groups were packed into the culture plates. The culture dishes were put in an incubator at $37 \mathrm{oC}$ for 48 hours. The distances of The Inhibition region of the three different materials employed in this study (group 1 , group 2 and group 3), were measured by Vernier calliper in millimeters unit around the specimens.

\section{Statistical analysis:}

Data was analyzed using one-way (ANOVA) followed by post-hoctest. The p-value $\leq 0.05$ was considered statistically significant. Statistical analysis was done using (SPSS) v.22 for windows. Mean and standard deviation values of fluoride release and inhibition zone were determined. 


\section{RESULTS}

\section{For fluoride release}

(Table 2 /Figure 2). Showed that the initial fluoride release of the Zirconomer group at day one revealed significantly $(\mathrm{P} \leq 0.05)$ the highest mean (32.674 \pm 0.922$)$ fluoride release value while specimens of Activa bioactive group at day 14 revealed significantly $(\mathrm{P} \leq 0.05)$ the lowest mean (11.633 \pm 0.562$)$ fluoride release value.

TABLE (2) The mean, standard deviation (SD) values of Fluoride release (ppm) of various groups at different time intervals.

\begin{tabular}{|c|c|c|c|c|}
\hline Groups & Day 1 & Day 7 & Day 14 & P-value* \\
\cline { 1 - 4 } Zirconomer & $32.674 \pm 0.922$ & $22.829 \pm 1.095$ & $17.465 \pm 14.303$ & \\
\cline { 1 - 4 } Cention n & $20.373 \pm 0.782$ & $16.869 \pm 0.945$ & $14.01 \pm 0.01$ & $\leq 0.05$ \\
\cline { 1 - 1 } $\begin{array}{c}\text { Activa } \\
\text { Bioactive }\end{array}$ & $18.61 \pm 1.09$ & $14.02 \pm 0.87$ & $11.633 \pm 0.562$ & \\
\hline
\end{tabular}

*One Way ANOVA.

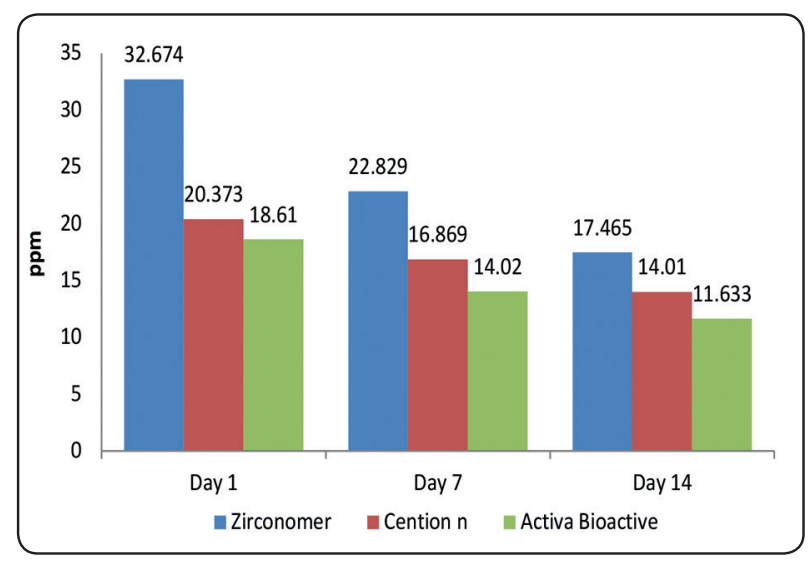

Fig. (2) Bar chart showing Mean and standard deviation of fluoride release (PPM) from various restorative materials at various time gaps

Regardless of the storage time whether day one, day 7 , or day 14 , Zirconomer revealed statistically significantly highest mean fluoride release value. This was followed by Cention-N. While, Activa bioactive group showed significantly lowest mean fluoride release. There was also, a significant variation in fluoride liberation value from group 2
(Cention $\mathrm{N}$ ) and group 3 (ACTIVIA Bioactive) at all the time gaps.

For each tested material; there was a statistically significant variation between different examined times at $\left(1^{\text {st }}, 7^{\text {th }}\right.$ and $14^{\text {th }}$ days $)$ where $(\mathrm{p} \leq 0.001)$. The highest mean value of fluoride release was in (Day 1), $\quad(32.674 \pm 0.922, \quad 20.373 \pm 0.782, \quad 18.61 \pm 1.09)$ for Zirconomer, Cention N, ACTIVIA Bioactive respectively. The least mean value of fluoride release was in (Day 14), (17.465 $\pm 14.303,14.01 \pm 0.01$, and 11.633 \pm 0.562 ) for Zirconomer, Cention N, ACTIVIA Bioactive-Restorative respectively.

\section{For antibacterial activity}

(Table 3/ Figure 3 and 4) showed that statistically significant largest inhibition zone versus Streptococcus mutans at 48 hours was detected with Cention $\mathrm{N}$ group (10.44 \pm 0.3090) followed by Zirconomer group (8.44 \pm 0.3019$)$. The smallest zone of inhibition observed with Activa bioactive group (7.24 \pm 0.3089$)$.

TABLE (3) Mean and standard deviation of inhibition zone between groups.

\begin{tabular}{|c|c|c|}
\hline Group & Mean \pm SD & P -value \\
\hline Zirconomer & $8.44 \pm 0.3019$ & \multirow{2}{*}{$<\mathbf{0 . 0 5}$} \\
\cline { 1 - 2 } Cention $n$ & $10.44 \pm 0.3090$ & \\
\cline { 1 - 2 } Activa BioACTIVE & $0.3089 \pm 7.24$ & \\
\hline
\end{tabular}

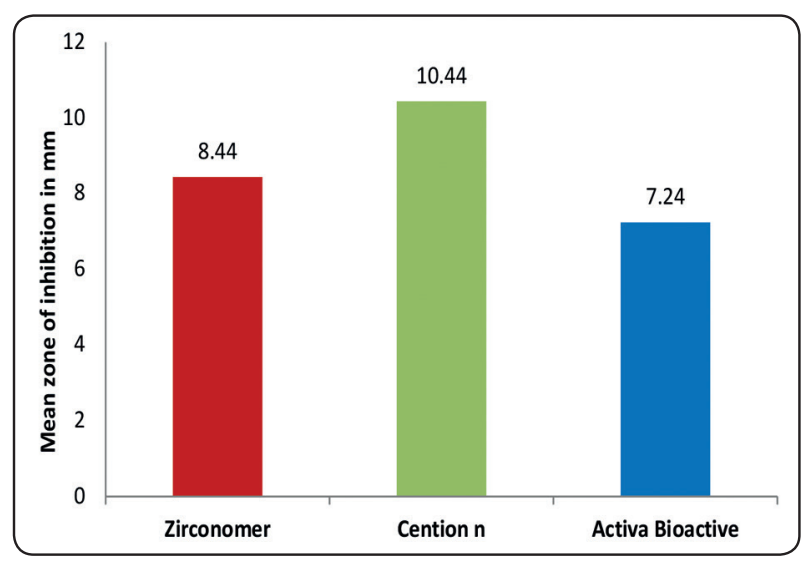

Fig. (3) Bar chart showing Mean and standard deviation of inhibition zone versus Streptococcus mutans at 48 hours of various restorative materials. 


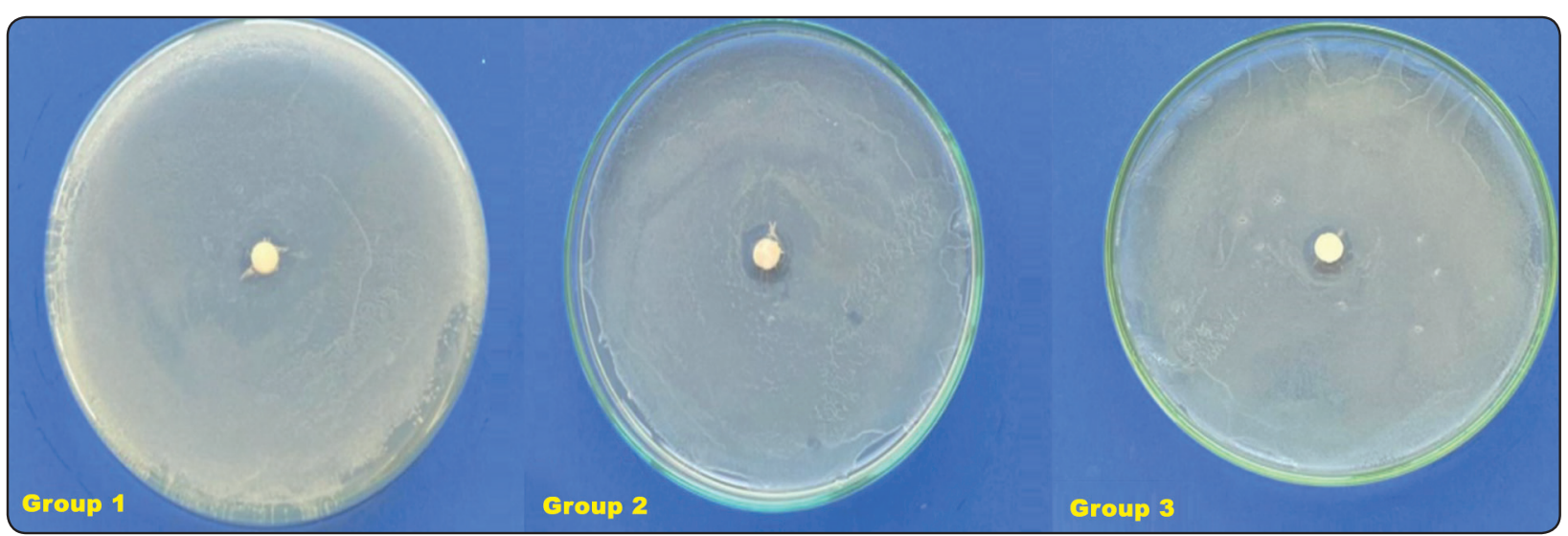

Fig. (4) Inhibition Zone versus Streptococcus mutans at 48 hours of various restorative material groups. (Group 1: Zirconomer group. Group 2: Cention group 3: ACTIVA BioACTIVE group)

\section{DISCUSSION}

Fluoride liberation from a glass ionomer is a complicated procedure and altered by internal and external elements. Internal element involving composition, liquid / powder balance, temperature, blending time, solvency, penetrability of the material, surface treatment, and finishing. External elements involving nature and $\mathrm{pH}$ of the media, investigational pattern, the ecological temperature and insightful measures investigative measures ${ }^{(13,14)}$.

Fluoride liberation from glass ionomer cements occurred by three methods: Superficial loss, diffusion across holes or fissures and body diffusion ${ }^{(15)}$.

This investigation was assumed to estimate and match the quantity of fluoride particle liberation from three diverse fluoride liberating restorative materials. The various materials in this investigation are designated for restoring a carious tooth in high and low stress bearing areas. ${ }^{(15)}$

In this investigation all examined fluoridereleasing materials liberated the most elevated quantity of fluoride particles on the 1st-day. Fluoride continued to be liberated in moderately little quantities from day 7 till day 14 .

The high quantity of fluoride liberation from Zirconomer group on the first day was possibly due to early burst of fluoride discharge from the glass components. The burst liberates due to the reaction of the Polyalkenic acid with the fluoride-containing glass elements during the setting reaction and also to the fast dissolution of fluoride from the external surface into the liquid. ${ }^{(16)}$

This was in concurrence with different an in -vitro investigations that have illustrated the same result in the first day. ${ }^{(16,17,13)}$

During the subsequent days the liberation of fluoride was decreased this may be due to reduce the dissolution of glass particles through cement pores and cracks ${ }^{(18)}$.

Alkasite material contains an alkaline-fillers, which creates acid-neutralizing particles. In its blended condition, alkaline glass represent $23.5 \%$ by weight, which liable for a generous quantity of fluoride discharge (4). The Findings of the existing investigation indicate that Cention $\mathrm{N}$ had fundamentally low fluoride liberation at all-time intervals when compared to Zirconomer. This related to that Cention $\mathrm{N}$ lacks a burst effect but constantly releases fluoride over the period may be due to a higher powder/liquid ratio and also a high amount of alkaline glass in its final state. This is in concurrence with Gupta et al (2019), who was reported that glass ionomers released more fluoride 
than Cention $\mathrm{N}$ at all intervals of measurements ${ }^{(19)}$. Also, this is in concurrence with Panpisut et al who compared resin modified glass ionomer with different contents and Cention $\mathrm{N}$ fluoride release, and they reported that cumulative fluoride release was at its highest in resin modified glass ionomer, then the second highest rate was in Cention $\mathrm{N}^{(20)}$.

ACTIVA Bioactive-Restorative in the current study had the lowest fluoride release at all-time intervals where it contains a patented, resilient resin matrix with energy-absorbing elastomeric components. This patented resin matrix might modify the permeability of this improved resin modified glass ionomer, leading to lower release capacity and recharged to acting as fluoride reservoir ${ }^{(21)}$.

Deionized (DI) water, saliva or $\mathrm{pH}$-cycling patterns are ideal methods to estimate the fluoride discharge from dental materials ${ }^{(22,23)}$. While saliva or $\mathrm{pH}$-cycling patterns could better mimic the oral conditions, deionized water was used in our study as a medium because it is simply available and shows well the fluoride liberation of the materials without the mistaking influence of minerals or organic molecules which might be presented in saliva or $\mathrm{pH}$-cycling solutions ${ }^{(22,23)}$. In addition, more fluoride is liberated in deionized water than in artificial saliva ${ }^{(23)}$.

Dental caries represents one of the most infectious diseases. It is a multi-factorial disease associated to the presence of cariogenic bacteria set in the dental plaque. In this study Streptococcus mutans microorganisms were selected as they are considered to be the most important group of bacteria initiating caries lesions. It was described that the number of salivary Streptococcus mutans in the oral cavity is related to the formation of fresh carious lesions, and it is generally accepted that decreasing the number of Streptococcus mutans also decreases caries activity ${ }^{(24,25)}$.

Antibacterial action of restorative materials was assessed versus Streptococcus mutans at
48 hours. Antibacterial properties of restorative materials have been estimated and the bactericidal influences are regularly due to their fluoride discharge. Different methods are included in the anti-cariogenic influences of fluoride on the teeth. Fluoride reduces construction of bacterial acids and glucans formed by Streptococcus mutans, which is recognized to remain the main causative element for carious lesions, and hence has a traditional application in examination the antimicrobial action of restorative materials ${ }^{(26)}$. In the existing study, the antibacterial action was assessed using the agar diffusion investigation. This permits bacteria to be investigated in a standard, reasonable, and simple technique for revealing the resistance ${ }^{(26)}$. In the current investigation biggest inhibition zone was detected with Cention $\mathrm{N}$ and the least with Activa Bioactive This variation in antimicrobial action could be associated with variation in liberation of fluoride and composition ${ }^{(27)}$.

In this study Cention-N presented high antibacterial activity as it in acidic $\mathrm{PH}$. due to presence of bacteria, released higher amounts of fluoride ion, the probable description is that Cention- $\mathrm{N}$ may responded more forcefully in the existence of acidic situation where possibly the surface unaffected layer may washed-out quicker, so baring the matrix for improved liberation of fluoride ions ${ }^{(5)}$.

Moreover, Cention-N revealed elevated alkalizing ability in acidic $\mathrm{pH}$. This could be owing to the hydroxyl and calcium ions liberate by alkaline glass fillers from Cention-N, that are capable to have exact influence on the $\mathrm{pH}$ levels, thus producing environments where the additional acidity owing to cariogenic bacterial activity could be counteracted ${ }^{(5)}$.

The bioactive materials Activa in this study presented weak antibacterial activity and considered as resin-based fluoride releasing materials as it has monomers such as urethane dimethacrylates. This weak antibacterial activity was reported due to release a smaller quantity of fluoride in comparison 
with Cention $\mathrm{N}$ and Zirconomer due to presence of urethane dimethacrylates and so, the less fluoride release may contribute to less antibacterial effect. We supposed that these changeable results could be related to variations in making the dental samples such as polymerization of the materials for example, the exact time of light-curing and how far the lightcure unit from the materials.

The limitations of this study include using standardized cylindrical specimens where in clinically the dimension of the cavity varies. In this study deionized water was used as a media to check fluoride release. In oral cavity saliva is the media. The existing investigation was operated in in-vitro conditions. Oral environment is active and altered from in-vitro conditions. Advance research can be conducted to check the recharge abilities of Cention $\mathrm{N}$.

\section{CONCLUSION}

Within the limitations, it tends to concluded that:

1. Zirconomer showed higher fluoride release than Cention N and ACTIVIA Bioactive-Restorative on all time intervals.

2. Cention $\mathbf{N}$ had a greater zone of inhibition, suggesting, better antibacterial activity than Zirconomer and ACTIVIA Bioactive-Restorative

Future studies should be directed towards controlled clinical trials with more complex experimental designs comprising of great number of reasons which affect the properties of dental materials in actual clinical conditions to draw valid conclusions.

\section{REFERENCES}

1. Ganss C, Lussi A. Erosive Tooth Wear: From Diagnosis to Therapy. Monographs in Oral Science. 2nd ed. Karger Publishers: Basel; 2012.pp. 22-31.

2. Forsten L. Fluoride release from a glass ionomer cement. Scand J Dent Res 1977; 85(6):503-4.

3. Dionysopoulos D, Sfeikos T, Tolidis K. Fluoride release and recharging ability of new dental sealants. Eur Arch Paediatr Dent 2016; 17(1):45-51.
4. Todd JC. Scientific Documentation. Cention N. Schaan, Liechtenstein: Ivoclar-Vivadent Press; 2016. pp. 1-58.

5. Bhattacharya S, Purayil TP, Ginjupalli K, Kini S, Pai S. Effect of thermocycling on the colour stability of aesthetic restorative materials: an in-vitro spectrophotometric analysis. Pesqui Bras Odontopediatria Clín Integr. 2020; 20:e5174

6. Croll TP, Berg JH, Donly KJ. Dental repair material: a resin modified glass-ionomer bioactive ionic resin-based composite. Compend Contin Educ Dent 2015; 36: 60-65.

7. Lassila L, Garoushi S, Vallittu PK, Säilynoja E. Mechanical properties of fiber reinforced restorative composite with two distinguished fiber length distribution. J Mech Behav Biomed Mater 2016; 60: 331-338.

8. Pameijer CH, Garcia-Godoy F, Morrow BR, Jefferies SR. Flexural strength and flexural fatigue properties of resin modified glass ionomers. J Clin Dent 2015;26(1):23-7.

9. Zirconomer Zirconia Reinforced Restorative. Available from http://www.shofu.com.sg/downloads/pdf/Zirconomer Brochure.pdf:

10. Patel MU, Punia SK, Bhat S, Singh G, Bhargava R, Goyal $\mathrm{P}$, et al. An in vitro evaluation of microleakage of posterior teeth restored with amalgam, composite and Zirconomer-A stereomicroscopic study. J Clin Diagn Res. 2015; 9: ZC65-7.

11. Abdulsamee N, Elkhadem AH. Zirconomer and Zirconomer improved (white amalgams): Restorative materials for the future. Review. EC Dent Sci. 2017; 15:134-50.

12. M.Jayanthi, P.John, J. Elizabetho, RSakthivel, E.Arun, S Girij, et al. Comparative evaluation of fluoride release and recharge of pre-reacted glass ionomer composite and nano-ionomeric glass ionomer with daily fluoride exposure: an invitro study. J Indian Soc Pedod Prev Dent. 2013;31(4):234-39.

13. Dhull KS, Nandlal B. Effect of low-concentration daily topical fluoride application on fluoride release of giomer and compomer: an in vitro study. J Indian Soc Pedod Prev Dent 2011; 29:39-45.

14. Guedes AP et al. Effect of Fluoride-Releasing Adhesive Systems on the Mechanical Properties of Eroded Dentin. Braz Dent J 2016; 27:153-9. 18

15. Tiwari S, Nandlal B. Effect of nano-filled surface coating agent on fluoride release from conventional glass ionomer cement: an in vitro trial. J Indian Soc Pedod Prev Dent 2013; 31; 91-5. 
16. Mungara J, Philip J, Joseph E, Rajendran S, Elangovan A, Selvaraju G. Comparative evaluation of fluoride release and recharge of pre-reacted glass ionomercomposite and nano-ionomeric glass ionomer with daily fluoride exposure: An in vitro study. J Indian Soc Pedod Prev Dent 2013; 31:234-9.

17. Walia R, Jasuja P, Verma KG, Juneja S, Mathur A, Ahuja L. A comparative evaluation of microleakage and compressive strength of Ketac Molar, Giomer, Zirconomer, and Ceram-x: An in vitro study. J Indian Soc Pedod Prev Dent 2016; 34:280-4.

18. Attar N, Önen A. Fluoride release and uptake characteristics of aesthetic restorative materials. J Oral Rehabl 2002;29(8):791-829.

19. Gupta, N., Jaiswal, S., Nikhil, V., Gupta, S., Jha, P., \& Bansal, P. Comparison of fluoride ion release and alkalizing potential of a new bulk-fill alkasite. Journal of Conservative Dentistry, 22(3), 296.

20. Panpisut, P., \& Toneluck, A. Monomer conversion, dimensional stability, biaxial flexural strength, and fluoride release of resin-based restorative material containing alkaline fillers. Dental Materials Journal, 39(4), 608-615.

21. Garoushi S, Vallittu PK, Lassila L. Characterization of fluoride releasing restorative dental materials. Dent Mater J 2018; 37:293-300.
22. Dionysopoulos D, Koliniotou-Koumpia E, HelvatzoglouAntoniades M, Kotsanos N. Fluoride release and recharge abilities of contemporary fluoride-containing restorative materials and dental adhesives. Dent Mater J 2013; 32:296-304.

23. Karantakis P, Helvatjoglou-Antoniades M, TheodoridouPahini S, Papadogiannis Y. Fluoride release from three glass ionomers, a compomer, and a composite resin in water, artificial saliva, and lactic acid. Oper Dent 2000; 25:20-5.

24. Marsh PD, N.B., The oral microflora and biofilms on teeth. In: Dental caries: the disease and its clinical management. Fejerskov O, Kidd EAM, editors. Oxford, UK: Blackwell Munksgaard, 2008: p. 163-187.

25. Marsh, P.D.,. Are dental diseases examples of ecological catastrophes? Microbiology, 2003. 149(Pt 2): p. 279-94.

26. Hotwani K, Thosar N, Baliga S, Bundale S, Sharma K. Antibacterial effects of hybrid tooth colored restorative materials against Streptococcus mutans: an invitro analysis. J Conserv Dent. 2013;16(4):319-22.

27. Shashibhushan KK, Basappa N, Subba Reddy VV. Comparison of antibacterial activity of three fluoridesand zinc-releasing commercial glass ionomer cements on strains of mutans streptococci: an invitro study. J Indian Soc Pedod Prev Dent. 2008;26 Suppl 2: S56-61. 